\title{
Une analyse économique des contrats de travail dans l'industrie du sport professionnel : Hold-up en football
}

An Economic Analysis of Labour Contracts in the Professional Sports Industry: Hold-up Problem in Football

Jérémie Bastien

Édition électronique
URL : http://journals.openedition.org/ei/6189

DOI : 10.4000/ei.6189

ISSN : 2553-1891

Éditeur

Association Économie et Institutions

Référence électronique

Jérémie Bastien, « Une analyse économique des contrats de travail dans l'industrie du sport

professionnel : Hold-up en football », Économie et institutions [En ligne], 27 | 2018, mis en ligne le 19 décembre 2018, consulté le 20 avril 2019. URL : http://journals.openedition.org/ei/6189 ; DOI

$10.4000 /$ ei.6189

Ce document a été généré automatiquement le 20 avril 2019.

Revue Économie et institutions 


\title{
Une analyse économique des contrats de travail dans l'industrie du sport professionnel : Hold-up en football
}

\author{
An Economic Analysis of Labour Contracts in the Professional Sports Industry: \\ Hold-up Problem in Football
}

Jérémie Bastien

L'auteur souhaite remercier les deux rapporteurs anonymes pour leurs remarques et commentaires. Il reste cependant seul responsable des erreurs et imperfections.

\section{Introduction}

1 En s'engageant avec le Paris Saint-Germain (PSG) en août 2017, Neymar da Silva Santos Júnior, dit Neymar, est devenu l'un des joueurs évoluant en Europe les mieux rémunérés avec un salaire brut annuel estimé à 52 millions d'euros, soit plus de 4,3 millions d'euros bruts par mois (environ 3 millions d'euros nets mensuels). En comparaison, le salaire brut moyen des footballeurs professionnels en Europe s'élève à plus de 46000 euros par mois. $\mathrm{Au}$ regard de ces niveaux de rémunération salariale, de nombreux travaux se sont consacrés à l'étude des conditions de fixation des salaires dans le football européen. L'intention est double : identifier les facteurs qui ont conduit à l'inflation généralisée des salaires ; et déterminer en quoi une infime fraction des joueurs perçoit des rémunérations bien supérieures à la moyenne.

Dans le premier cas, un consensus scientifique se forme dans l'explication de l'inflation salariale du football européen par le recours à l'hypothèse de "course à l'armement » . Brièvement, elle considère que, sous l'impulsion de nouveaux capitaux, les clubs ont intensifié leur concurrence dans l'obtention des joueurs à partir des années 1980, créant une véritable course à l'armement qui a pesé à la hausse sur les salaires. Cette course à 
l'armement s'est amplifiée après l'arrêt Bosman - qui a consacré la libéralisation du marché du travail dans le sport professionnel en Europe - et a ainsi contribué à l'envolée des salaires, d'une part, et à la croissance du prix des transferts (Brocard 2015) d'autre part, les clubs surenchérissant les uns sur les autres afin de recruter les meilleurs joueurs. Cette dynamique concurrentielle a été permise par la contrainte budgétaire «molle » des clubs . Conformément à celle-ci, les dépenses salariales et de recrutement ne sont pas contraintes par le respect d'un équilibre budgétaire du fait de la récurrence des apports financiers ex post (i.e. en fin de saison) des actionnaires et des télévisions. Les clubs bénéficient par ailleurs d'un système de crédit ainsi que d'un régime fiscal souples qui les autorisent à des niveaux d'endettement bancaire et d'arriérés de paiement élevés soutenant in fine leurs dépenses.

Dans le second cas, bien qu'elles se penchent rarement de manière exclusive sur le football, les études quant aux hautes rémunérations des vedettes du sport professionnel se multiplient. Nombre d'entre elles s'appuient sur la théorie des superstars développée par Sherwin Rosen . Elle conduit au résultat stipulant que de petites différences de talent engendrent d'importants écarts de rémunération, ce qui s'explique par la rareté relative du talent et par la taille du marché du talent. Par ce résultat, la théorie du tournoi, appliquée au sport (Rosen 1986) à partir des travaux de l'école du public choice (Tullock 1980), n'est pas étrangère à la théorie des superstars. Dans son application privilégiée aux sports professionnels individuels, elle énonce que la performance sportive des athlètes est conditionnée par les gains qu'ils retirent des différents paliers de la compétition. Ainsi, les joueurs sont incités à être davantage performants plus les dotations financières sont élevées et plus l'écart de gains est important, i.e. lorsque les revenus se concentrent sur les premières places de la compétition . D'autres études cherchent à dépasser la théorie standard de la productivité marginale pour rendre compte des salaires des vedettes du sport professionnel. À l'appui de la théorie du salaire d'efficience, Patrice Bouvet explique par exemple que les hauts salaires des superstars des sports par équipe s'apparentent à des «salaires d'appel ». Ils constituent des rémunérations suffisamment élevées pour que les dirigeants de clubs puissent recruter et conserver les meilleurs joueurs, voire afin de les inciter à de meilleures performances sportives. Dans leur application spécifique au football professionnel, ces travaux sur le niveau de la rémunération des superstars soulignent par ailleurs que le marché du travail est segmenté (Bourg 1983, 1988, 1990, 1998) à partir de la théorie du dualisme (Doeringer \& Piore 1971). D’un côté, le segment supérieur du marché du travail (marché primaire) regroupe les meilleurs joueurs qui bénéficient de très hautes rémunérations salariales et, de l'autre, le segment inférieur (marché secondaire) rassemble l'ensemble des autres joueurs pour qui les salaires sont plus modérés bien que demeurant élevés.

4 Si à la fois l'hypothèse de course à l'armement et la théorie des superstars nous apparaissent pertinentes, ces deux approches ne permettent pas de restituer les relations de pouvoir entre les clubs de football et les joueurs professionnels, ces derniers disposant d'un rapport de force favorable qui leur permet de percevoir d'importantes rémunérations. C'est pourquoi, en complément de ces deux hypothèses, nous proposons une nouvelle approche afin de rendre compte de l'inflation du coût des footballeurs professionnels en Europe à la lumière des jeux de négociations entre les acteurs. Dans cette perspective, nous recourons au « problème du hold-up » qui, au sein des approches contractuelles de la firme qui caractérisent la nouvelle microéconomie (Coriat \& Weinstein 2010) telle qu'elle s'est principalement développée à partir des travaux sur les 
coûts de transaction (Coase 1937; Williamson 1971) et sur les contrats incomplets (Grossman \& Hart 1986; Hart 1995), est un élément central de l'étude des relations interindividuelles. Si l'analyse du hold-up s'est à l'origine constituée au regard des relations contractuelles entre firmes indépendantes, elle s'est ensuite étendue à l'étude des relations intra-entreprise, et plus particulièrement à l'étude des relations salariales .

Dans ce contexte, l'objectif de l'article est d'étudier les relations contractuelles entre les clubs et les joueurs au regard des processus de négociation qu'elles impliquent. En élargissant l'étude à l'ensemble des segments du marché du travail, i.e. sans nous focaliser sur les superstars, nous nous interrogeons sur la capacité des joueurs à capter une large part des richesses générées par le football professionnel européen. L'idée principale est que les joueurs installent un tel pouvoir de négociation dans leur rapport au club qu'ils font naître une situation de hold-up lors des périodes de renégociation contractuelle : soit ils «braquent » effectivement leur club en obtenant un renouvellement de leur contrat avec augmentation du salaire, soit ils obtiennent un salaire plus élevé dans un autre club.

6 Après être revenu sur la théorie du hold-up et après avoir passé en revue ses développements empiriques dans une première partie, l'article appréhende, dans une deuxième partie, les fondements et les conséquences du problème du hold-up dans le football professionnel européen à travers la relation contractuelle spécifique entre un club et un joueur. La troisième partie se concentre sur les stratégies développées par les clubs face au risque du hold-up, la principale étant l'augmentation des indemnités de transfert, ce qui contribue en dernier ressort à amplifier leurs déséquilibres financiers.

\section{Le problème du hold-up}

\subsection{L'analyse théorique du hold-up}

7 Les approches économiques néo-institutionnalistes s'appliquent à rendre compte des modes d'organisation (Ménard 2008) des échanges au regard des relations contractuelles qui les caractérisent. En effet, les contrats sont l'expression de la codification des rapports bilatéraux inhérents à tout mode de coordination entre des agents individuels. L'analyse des contrats est fondamentale en ce que les relations contractuelles sont systématiquement sources de coûts : au-delà des contrats qui résultent de la coordination des agents sur le marché (Coase 1937; Williamson 1971), la coordination au sein de l'entreprise génère elle-aussi des coûts de transaction, étant donné que la firme représente un nœud de contrats (Alchian \& Demsetz 1972 ; Jensen \& Meckling 1976) ${ }^{1}$. Dès lors, toute structure de gouvernance se définit par un régime contractuel spécifique lui étant associé.

Quel que soit le mode d'organisation étudié, le "problème du hold-up " naît d'une situation contractuelle dans laquelle deux agents collaborent afin d'en tirer des avantages réciproques. Ils dégagent un surplus de cette relation contractuelle qui est appelé rente organisationnelle. La valeur de cette rente est conditionnée par la collaboration des deux parties au contrat puisque chacun des cocontractants n'aurait pu atteindre un tel surplus seul. Quand cette valeur atteint son maximum à la condition d'un usage particulier qui ne doit pas être détourné , l'échange produit un actif spécifique qui souligne la dépendance des parties au contrat. Comme le stipule la théorie du partage de la rente, il est donc légitime que chacune des parties perçoive une fraction du surplus généré . Le partage de 
la rente est déterminé dans les termes du contrat qui sont négociés ex ante entre les deux parties. La négociation précontractuelle est donc essentielle.

Toutefois, comme les agents collaborent dans un environnement d'incomplétude de l'information, les contrats tels qu'ils ont été édictés et négociés en amont ne peuvent $a$ priori pas prévoir l'ensemble des situations auxquelles la relation transactionnelle pourrait donner lieu. Par conséquent, les contrats sont eux-aussi incomplets (Grossman \& Hart 1986; Hart 1995), ce qui incite les cocontractants à développer des comportements opportunistes post-contractuels dont l'objectif est d'obtenir une part plus importante de la rente. L'un des cocontractants, profitant d'une asymétrie stratégique qui le place en position de force, cherche à tirer profit de la transaction. Une fois le contrat conclu, il développe ainsi des stratégies visant à stimuler une renégociation des termes du contrat dans le but d'instaurer un partage de la rente lui étant plus avantageux. Il s'approprie à terme une quasi-rente, appelée quasi-rente appropriable ou quasi-rente relationnelle , ou encore quasi-rente organisationnelle. Il y a alors hold-up car le surplus provenant de l'échange contractuel est accaparé par une partie au détriment de l'autre. cocontractants dans le but de constituer un actif spécifique sont élevés. Plus précisément, les investissements collectifs qui surviennent dans le cadre du contrat rendent une des parties si spécifique qu'elle en devient irremplaçable. Elle est indispensable à la constitution d'un actif spécifique pour l'autre cocontractant. On peut alors parler d'actif cospécialisé . La valeur liée à l'investissement dans de tels actifs est volatile dans la mesure où elle dépend du comportement des parties au contrat. En ce sens, le cocontractant s'étant rendu indispensable dispose d'un pouvoir de négociation élevé visà-vis de l'autre cocontractant à partir duquel il va chercher à s'octroyer une quasi-rente. Pour y parvenir, les stratégies de retrait sont privilégiées par les agents étant en position dominante. Il s'agit par exemple de menacer d'une rupture anticipée de contrat ou d'annoncer par avance une éventuelle non-reconduction du contrat à son terme. Dans ce cas, par crainte de voir disparaître la rente - et les investissements sous-jacents - en même temps que le départ de son cocontractant, l'autre partie au contrat peut être amenée à renégocier les clauses du contrat dans le sens des attentes du cocontractant qui menace d'un retrait. Il y a donc hold-up puisque le cocontractant en position de force est parvenu à s'approprier une part supplémentaire du surplus généré collectivement. Dès lors, "[...] l'investissement dans un actif spécifique [...] met son détenteur dans une position de faiblesse relationnelle et de dépendance à l'égard des personnes avec lesquelles il mène des échanges" .

11 Le pouvoir de négociation de la partie indispensable à la constitution d'un actif spécifique est renforcé en présence d'actifs redéployables. Elle détient alors la capacité de transférer les actifs spécifiques d'un contrat à l'autre, tout en ayant profité des investissements collectifs des précédentes relations contractuelles. Concrètement, cette partie au contrat est en mesure de redéployer son activité ailleurs grâce à ses compétences ainsi qu'aux compétences qu'elle a acquises dans le précédent contrat. Elle est à même d'engendrer dans une nouvelle collaboration contractuelle un actif spécifique semblable à celui produit dans une collaboration antérieure. Cette transférabilité des actifs est d'autant plus problématique que l'agent quitte un contrat pour une activité de nature similaire. En définitive, la présence d'actifs redéployables apporte de la crédibilité aux stratégies de retrait développées par les agents. Au-delà du fait de subir une dévalorisation du contrat provoquée par la disparition de la rente organisationnelle, un 
des cocontractants supporte en quelque sorte une fuite de ses investissements spécifiques lorsque l'autre partie quitte la relation contractuelle pour reproduire son activité ailleurs. Le rapport de force est donc largement au bénéfice de la seconde partie, la première étant contrainte de renégocier en acceptant les termes réclamés afin de préserver la rente organisationnelle.

Dans la continuité des travaux constitutifs de la théorie des coûts de transaction, le cadre d'application privilégié du problème du hold-up a d'abord concerné les rapports interentreprises, à commencer par les relations entre une firme et son fournisseur (Klein et al., 1978). Les développements néo-institutionnalistes plus récents ont ensuite souligné la pertinence du processus de hold-up pour l'étude des relations contractuelles entre un employeur et son salarié et qui sont codifiées par un contrat de travail, plus particulièrement lorsqu'un salarié devient déterminant dans la constitution d'un actif spécifique. Autrement dit, la rente née de la collaboration contractuelle est conditionnée par la présence du salarié parce qu'il détient des savoirs et savoir-faire difficilement reproductibles (l'interchangeabilité des salariés est faible et limitée). D'autant plus que les salariés profitent d'investissements collectifs réalisés par l'entreprise qui cherche à renforcer un actif spécifique. Les dépenses de formation du personnel en sont un bon exemple. Par conséquent, les salariés possèdent la capacité de confisquer des actifs clés en tant qu'actifs collectifs de l'entreprise. Ils détiennent en cela des droits de propriété implicites ou quasi-droits de propriété sur ces actifs. Ils sont alors en position de force dans leur rapport à l'employeur et tentent d'en tirer parti en menaçant de quitter l'entreprise. Dans ce cas, l'employeur peut être contraint de renégocier les termes du contrat à l'avantage des salariés car le départ de ses salariés « spécifiques » contribuerait à dévaloriser l'actif. L'employeur se fait "braquer" par ses employés puisque les dispositions relatives au partage de la rémunération de l'actif spécifique évoluent au bénéfice des seconds. De par leur importance dans la chaîne de valeur de l'entreprise, ces salariés profitent d'une "renégociation par effet de levier» (ibid.) qui leur permet de capter les richesses et de percevoir une rémunération élevée.

Le problème du hold-up crée donc un véritable contexte d'incertitude informationnelle à l'origine de situations d'asymétries d'information. La conséquence en est que, en période précontractuelle, parce qu'ils anticipent l'opportunisme post-contractuel de la partie adverse visant à s'adjuger le bénéfice de la transaction, les cocontractants peuvent faire le choix de limiter leurs investissements afin de se prémunir d'une situation de dépendance vis-à-vis de l'autre partie . L'objectif sous-jacent est de limiter les périodes de renégociation pouvant être désavantageuses. Le résultat immédiat en est une situation de sous-optimalité en raison de la diminution des investissements dans les actifs spécifiques. Les investissements sont plus faibles que ceux qui auraient été réalisés par les cocontractants en l'absence des craintes de hold-up.

\subsection{Les approches empiriques du hold-up}

Peu de travaux empiriques viennent appuyer ce résultat théorique, plus particulièrement quant à la sous-optimalité des investissements en raison du hold-up. Ce défaut quantitatif s'explique essentiellement par le fait que les agents cherchent à se prémunir des conséquences néfastes du hold-up (Malcomson 1997; Williamson 1985). Les études expérimentales démontrent plutôt comment les agents réagissent, i.e. les solutions qu'ils 
mettent en place face au problème du hold-up. La partie qui anticipe ex ante un risque de hold-up peut ainsi recourir à deux stratégies précontractuelles.

La première consiste à allonger la durée du contrat . Un contrat de long terme limite les comportements opportunistes par la réduction des éventuelles périodes de renégociation. De même, cette extension de la durée des contrats permet de figer sur une plus longue période les termes de la relation contractuelle. L'exemple le plus significatif est la fixation d'un prix sur du long terme entre une entreprise et son fournisseur afin d'empêcher ce dernier, qui s'est rendu irremplaçable, de réviser ses prix à la hausse en période postcontractuelle. Cette solution est notamment illustrée par Paul Joskow à travers l'exemple des centrales thermiques électriques étasuniennes approvisionnées par les mines de charbon à proximité.

La deuxième stratégie est l'intégration verticale (Williamson 1971), le contrôle instauré par la coordination administrative permettant de réduire l'opportunisme des parties au contrat. Cette solution repose à nouveau sur l'exemple-type d'une firme et de son fournisseur. L'entreprise absorbe l'entité qui l'approvisionne afin de ne pas être dépendante des aléas qui pourraient l'affecter et donc conduire à une situation de hold-up. Au-delà du fait de ne plus être soumise aux variations des prix du fournisseur, la firme se préserve des problèmes d'approvisionnement potentiels. La résolution des problèmes d'approvisionnement par le recours à l'intégration verticale a été démontrée par Benjamin Klein et al. dans le cas de l'industrie automobile à partir de l'exemple de l'acquisition du fournisseur de carrosserie Fisher Body par le constructeur américain General Motors dans les années $1920^{2}$.

Parmi les travaux empiriques sur le hold-up, deux ont plus particulièrement retenu notre attention.

18 En raison des similitudes existantes avec notre objet d'étude, il s'agit tout d'abord d'une analyse de l'industrie financière . L'auteur y démontre la présence d'un processus de holdup dans la relation qu'entretiennent les traders avec les banques qui les emploient: comme ils disposent d'un fort pouvoir de négociation, les traders sont en mesure d'obtenir de hautes rémunérations, notamment grâce à l'augmentation de la part variable de leur rémunération (les bonus). Afin d'ancrer leur pouvoir, ils développent des stratégies de retrait légitimées par la présence d'une forte concurrence et par une conjoncture favorable. Parce qu'ils détiennent des aptitudes, une technique ainsi qu'une clientèle hautement spécifiques, de surcroît enrichies par les investissements des banques, tout départ de traders d'une banque entraine un double effet: (a) une diminution de la valeur de l'activité pour laquelle ils étaient employés (condition de spécificité) ; (b) un transfert

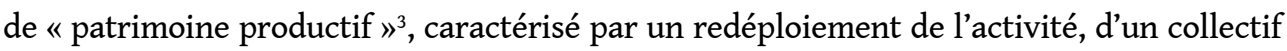
et d'un portefeuille de clients chez un concurrent (condition de transférabilité). Cette double conséquence incite les dirigeants des banques à conserver leurs traders en leur offrant d'importantes revalorisations salariales. Il y alors hold-up des traders.

Le deuxième travail empirique que nous soulignons plus expressément est celui mené par Meng-Chi Tang dans la mesure où il s'intéresse au sport professionnel. L'auteur y teste la relation théorique entre la durée des contrats et le niveau des investissements spécifiques dans le cas de la National Football League nord-américaine. En se focalisant sur les contrats des nouveaux joueurs qui entrent dans la ligue (i.e. issus de la draft), l'auteur démontre que les franchises (i.e. les clubs) tendent à augmenter la durée des contrats des joueurs quand leur recrutement nécessite un niveau important d'investissements spécifiques. 
Cette méthode a pour but de se prémunir des effets d'une situation de hold-up. L'auteur précise cependant que la durée des contrats des joueurs reste conditionnée à la fois par le surplus espéré lors d'une embauche et par le niveau de rémunération des joueurs.

\section{Hold-up en football}

\subsection{Une relation contractuelle singulière : de la valeur des joueurs à la reconnaissance d'actifs spécifiques et transférables}

En nous appuyant simultanément sur les approches théoriques et empiriques dont nous venons de faire état, nous appliquons le problème du hold-up à la relation contractuelle de travail entre un club (l'employeur) et un joueur (l'employé) dans le football professionnel européen de première division, en postulant qu'un surplus naît de ce type de contrat. Contrairement à une grande majorité de secteurs, les contrats y sont des contrats à durée déterminée. Ils se sont substitués aux contrats «à vie » dans les années 1970. Ces derniers nécessitaient obligatoirement l'autorisation des dirigeants pour qu'un joueur sous contrat puisse quitter un club pour un autre. Un système de réservation analogue au modèle nord-américain (clause de réserve) était alors en fonction. Un club pouvait ainsi conserver un joueur durant toute la durée de sa carrière, limitant de fait son pouvoir de négociation et le rendant « esclave » du club . L'apparition du contrat à durée déterminée a profondément modifié les logiques de fonctionnement du football professionnel en Europe. Son introduction a accordé aux joueurs une certaine liberté contractuelle (droit de quitter un club au terme d'un contrat) qui a notamment mené à l'accroissement des mutations de joueurs entre clubs. Par ailleurs, dès 1995, en vertu de l'arrêt Bosman, la compensation financière de fin de contrat a été supprimée. Cela signifie qu'un joueur peut librement quitter un club pour un autre au terme de son contrat, et qu'un club a la possibilité d'acquérir un joueur gratuitement dès lors qu'il est en fin de contrat. La mobilité fut d'autant plus favorisée que cette jurisprudence a également mis fin aux quotas nationaux. Depuis lors, les clubs peuvent recruter sans limite les joueurs étrangers vers leur territoire national à condition que ces derniers fassent partie de la communauté sportive européenne ${ }^{4}$.

21 À partir des années 1970, le rapport de force entre les clubs et les joueurs s'est donc progressivement inversé au bénéfice des seconds (Brocard 2010), les joueurs se constituant un véritable pouvoir de négociation dans leurs relations aux propriétairesdirigeants de clubs. Ce pouvoir des joueurs s'explique avant tout par le fait qu'ils se révèlent indispensables à la constitution d'actifs spécifiques pour les clubs. En effet, tout recrutement ou tout renouvellement d'un contrat s'effectue dans la finalité d'améliorer la productivité du club. Les joueurs sont donc fondamentaux dans la valorisation des clubs. Un intérêt partagé émerge de l'échange puisque les clubs s'assurent des meilleurs sportifs possibles et les joueurs perçoivent un salaire qu'ils jugent élevé . Dans cette perspective, les politiques de recrutement sont basées sur un ensemble de prévisions quant à la triple valeur des joueurs.

En premier lieu, les clubs anticipent la valeur d'usage des joueurs. Il s'agit de prévoir les performances on-the-field des joueurs, et plus précisément de déterminer ce que peuvent être leurs effets sur les flux de revenus des clubs (gains sportifs). Par conséquent, parce qu'ils opèrent en ligue ouverte (Andreff 2007), les clubs cherchent à maximiser les victoires en constituant une équipe performante par le recrutement des meilleurs joueurs 
possibles à leur poste. À ce titre, par leurs compétences techniques, les footballeurs sont identifiés comme un facteur rare pour trois raisons : (1) une substituabilité limitée entre postes ; (2) une performance individuelle conditionnée par la performance collective ; (3) et une complexité tant sportive que financière à effectuer des remplacements et des recrutements poste pour poste.

En deuxième lieu, les clubs anticipent la valeur de non-usage des joueurs. L'objectif est d'évaluer la capacité d'attractivité des joueurs pour les financeurs des clubs. Autrement dit, les clubs tentent de déterminer si la présence d'un joueur sera génératrice d'un accroissement de l'intérêt de certains financeurs (sponsors, spectateurs, médias, etc.) pour le club, ce qui se traduirait par un flux de revenus supplémentaire. Les clubs cherchent donc à recruter des joueurs pour qui l'intérêt des financeurs est important, i.e. qui sont en mesure de contribuer à l'accumulation du club à travers leur image. Dans ce cas, les performances off-the-field des joueurs sont fondamentales, même si les performances purement sportives semblent aussi contribuer au pouvoir marketing des joueurs (le "faire vendre »). Cette politique de recrutement orientée vers les joueurs bankable va dans le sens de la maximisation des revenus des clubs ${ }^{5}$. Au-delà du cas de la star qui génère d'importantes ventes de maillots à son effigie, l'exemple significatif de ce type de pratiques concerne le recrutement de joueurs étrangers, ces derniers permettant aux clubs de pénétrer de nouveaux marchés géographiques (comme le continent asiatique).

24 En troisième et dernier lieu, les joueurs sont primordiaux pour les clubs car ils pèsent sur leur valeur fondamentale en tant qu'actifs intangibles. En fait, les clubs affectent les contrats de joueurs à l'actif de leur bilan . Cette pratique comptable innovante et spécifique au football professionnel permet aux clubs de gonfler leurs actifs.

La valeur des clubs est donc largement conditionnée par l'activité des joueurs qui les composent. Ces derniers sont les acteurs clé de la valeur économique des clubs, ce qui leur octroie un pouvoir de négociation élevé duquel ils cherchent légitimement à tirer avantage. C'est dans cette optique que les joueurs développent des stratégies postcontractuelles afin de s'accaparer une partie plus importante de la rente qu'ils ont largement suscitée. Les menaces de départ sont utilisées en ce sens. En effet, tout départ de joueur entraîne de facto la disparition d'une rente organisationnelle, étant donné qu'une relation productive spécifique avec un club cesse. Le surplus qui est apparu lors de la collaboration contractuelle disparait avec son interruption. Le retrait d'un joueur constitue en quelque sorte un manque à gagner pour le club .

Les retraits de joueurs sont d'autant plus problématiques que les clubs y consentent de lourds investissements dans le but de constituer et de renforcer un actif spécifique à travers le développement de leur capital humain. Il s'agit tout d'abord des dépenses des clubs dans la formation, l'entretien et le perfectionnement des joueurs . Il s'agit aussi des salaires versés aux joueurs, puisqu'ils financent indirectement l'accumulation d'un savoir-faire dans la pratique de compétition, i.e. on the job training . Ces investissements fragilisent les clubs dans leur relation de pouvoir aux joueurs et renforcent le risque de hold-up : le départ d'un joueur entraîne la disparition de la rente, d'une part, mais aussi de l'ensemble des dépenses qui ont été réalisées dans l'optique de dégager ce surplus, d'autre part. En conséquence, les clubs peuvent être contraints de renégocier les termes du contrat à leur défaveur, notamment par une augmentation de la rémunération des joueurs, d'où la réalisation effective du hold-up. 
27 Sur ce point, il est important de préciser que les superstars sont à l'origine d'actifs hautement spécifiques par leurs valeurs d'usage, de non-usage et comptable. Pour cette raison, leur pouvoir de négociation est très élevé dans la mesure où ils sont indispensables pour les clubs, notamment de par leur forte valeur de non-usage. Ce pouvoir s'explique par une substituabilité limitée (rareté) sur ce segment du marché du travail qualifié de " micro-marché particulier». Conformément à la théorie du partage de la rente, il est donc légitime que ces vedettes perçoivent une large partie de la rente qu'elles ont suscitée, ce qui explique leur rémunération salariale élevée, à l'image des salaires bruts annuels de Lionel Messi (36 millions d'euros), de Cristiano Ronaldo (32 millions d'euros) ou encore de Neymar (20 millions d'euros) en 2016. Ce type de joueurs saisit ainsi une véritable rente de situation conduisant au winner-takes-all-model.

Pour autant les superstars ne possèdent pas l'exclusivité de la constitution d'actifs spécifiques. De notre point de vue, le degré de spécificité des actifs revêt une certaine forme de subjectivité. Nous entendons par là que des joueurs peuvent se révéler tout aussi indispensables que des superstars dans des clubs de moindre importance que les grands clubs européens. À titre d'exemple, s'il est incontestable que Neymar ou qu'Edinson Cavani sont indispensables au PSG, tant du point de vue sportif que financier, Malcom et Ivan Santini le sont respectivement tout autant pour les Girondins de Bordeaux et le Stade Malherbe de Caen, tout comme l'était André Ayew lorsqu'il évoluait à l'olympique de Marseille (OM) par ses performances sportives et sa capacité à "faire vendre ». Au regard de cette hypothèse du degré de spécificité des actifs, le pouvoir de négociation élevé qui conduit à la situation de hold-up n'est donc pas l'exclusivité du marché primaire du travail. Des joueurs issus du marché secondaire peuvent tout à fait disposer d'un rapport de force à leur avantage et en tirer parti, ce que confirme le niveau relativement élevé des salaires moyens dans le football. Toutefois, l'hétérogénéité du marché du travail est telle que des joueurs issus du segment inférieur ne détiennent aucun pouvoir de négociation et perçoivent de faibles rémunérations.

Dans son application au football, le problème du hold-up est par ailleurs renforcé par la condition de transférabilité des actifs. Les joueurs sont capables de reproduire leur activité d'un club à l'autre, alors même que celle-ci a été entretenue voire perfectionnée sous le financement du ou des précédents clubs. Les joueurs peuvent ainsi transférer des actifs spécifiques d'une relation contractuelle à une autre. Ils s'approprient de la sorte des quasi-droits de propriété sur les actifs collectifs d'un club, et ils peuvent les redéployer dans un autre club. Dès lors, au-delà de la perte d'une rente organisationnelle, les clubs constatent la reproduction de cette rente dans une autre relation contractuelle, le niveau de cette rente étant au demeurant fonction des investissements spécifiques des clubs antérieurs. Les clubs subissent d'une certaine façon une fuite de leurs investissements spécifiques7. Cette transférabilité des actifs s'exprime essentiellement via la valeur d'usage des joueurs. Toutefois, de par leur valeur de non-usage, les départs de joueurs peuvent engendrer une fuite de certains financeurs. C'est plus particulièrement le cas des superstars. Ce type de joueurs se substitue de plus en plus aux clubs en termes d'attractivité, le football étant influencé par un processus plus global d'individualisation et de déterritorialisation. Certains financeurs, dont les (télé)spectateurs et les sponsors, sont alors davantage fidélisés à un individu (i.e. un joueur) qu'à un club. À l'instar des grands financiers, les superstars du football sont des « rainmakers " puisqu'ils sont capables d'emporter avec eux, d'une entreprise (club) à l'autre, des clients. De la sorte, indirectement, en permettant aux joueurs de pérenniser leur activité et de renforcer 
leurs capacités, les clubs financent cette accumulation en termes d'image qui sera redéployée dans un autre club. Cette condition de transférabilité est davantage problématique lorsque le joueur rejoint un club concurrent. Dans cette perspective, audelà d'être un marché de personnes, le marché du travail des footballeurs professionnels est aussi un marché de transferts d'actifs, i.e. de ce que l'individu emporte avec lui ${ }^{8}$.

\subsection{L'opportunisme des joueurs et les conséquences du problème du hold-up} (prestige, dépassement de soi, culte de soi, etc.), l'aspect financier est central dans les stratégies développées par les sportifs de haut niveau (Bourg 2008). Dans le football, audelà de satisfaire l'objectif de maximisation des revenus des joueurs, cette motivation financière s'explique aussi par le fait que le salaire constitue un signal pour les recruteurs . Ainsi, afin de gagner l'intérêt des meilleurs clubs, les joueurs intègrent l'aspect salarial à leur stratégie de réputation'. Dans cette optique, ils s'appuient sur leur pouvoir de négociation vis-à-vis des clubs afin de peser à la hausse sur leur rémunération salariale. Pour cela, ils ont pour objectif intermédiaire de multiplier les périodes de renégociations contractuelles afin qu'elles aboutissent à une revalorisation salariale. La constitution de contrats de courte durée ou l'instauration de clauses de renégociation salariale aux contrats sont deux outils permettant de satisfaire cet objectif intermédiaire. Les stratégies de retrait sont des stratégies plus offensives - et couramment adoptées - qui visent à ouvrir des périodes de renégociation en cours de contrat.

cadre concurrentiel dans lequel se développent ces stratégies est primordial. Dans le cas des contrats de travail, le problème du hold-up se renforce sous l'effet de l'intensité concurrentielle. Les menaces de départ gagnent en légitimité avec le nombre d'employeurs potentiels. C'est pourquoi les stratégies de joueurs ont d'autant plus de chance d'atteindre leur finalité qu'elles reposent sur des "points d'appui». Lors d'une renégociation, les joueurs sont donc incités à signaler l'intérêt d'un ou plusieurs clubs. Le contexte actuel du football européen - dopé par les dépenses des clubs "émergents» dont le PSG et Manchester City - contribue à renforcer ces points d'appui, à la fois en augmentant le nombre d'employeurs potentiels et en pesant à la hausse sur les salaires. Dans cet environnement, les joueurs, à l'aide de leur agent sportif, cherchent à exploiter l'imperfection de l'information . Ils peuvent simuler l'intérêt de clubs concurrents afin de renforcer leur stratégie de retrait et surtout afin que le hold-up se réalise effectivement ${ }^{10}$. Les joueurs profitent ainsi d'asymétries d'information dans lesquelles le rôle de la rumeur, véhiculée par les médias, ne doit pas être négligé.

En outre, le cadre temporel dans lequel s'inscrit la stratégie de retrait (le timing) est fondamental . Bien que les menaces de départ de joueurs ne se révèlent concrètes qu'à partir de l'ouverture des périodes réglementaires de transferts, i.e. lors des mercato estival et hivernal qui limitent de fait l'activité du marché des transferts à deux périodes, le choix du moment où le joueur va développer sa stratégie de retrait reste déterminant. Il convient d'identifier une période cruciale pour le club. Par exemple, la menace de hold-up sera d'autant plus crédible qu'elle intervient dans le même temps que le départ avéré d'autres joueurs, dans le cas d'une qualification pour une compétition européenne la saison à venir, ou encore dans le cas d'une future entrée en bourse. 

stratégies de retrait qu'ils développent conduit donc à une situation de hold-up. Celle-ci se traduit par deux issues pour lesquelles l'aboutissement est similaire, à savoir l'augmentation des salaires des joueurs. La première hypothèse est que le club décide de conserver un joueur en lui proposant un nouveau contrat assorti d'une revalorisation salariale. Dans ces circonstances, il y a réalisation effective du hold-up. La seconde hypothèse repose sur le départ concret du joueur pour un autre club qui lui propose de meilleures conditions contractuelles et salariales. Chaque saison, de nombreux exemples illustrent et valident ces deux hypothèses.

Pour la première, nous pouvons notamment mentionner les stratégies de retrait mises en place par Sergio Ramos et par Thiago Motta à l'été 2015. Alors qu'ils étaient encore sous contrat, tous deux ont engagé à cette période un processus de renégociation avec leur club respectif (i.e. le Real Madrid et le PSG) en s'appuyant simultanément sur : (i) leur contribution aux performances sportives (et financières) du club; (ii) leur palmarès sportif ; (iii) l'intérêt de clubs concurrents (des points d'appui), à savoir Manchester United dans le cas de S. Ramos et l'Atletico Madrid dans le cas de T. Motta. Dans le cadre de sa stratégie de retrait, ce dernier a par ailleurs déclaré publiquement à quelques jours de la clôture du mercato estival : «Je vois les responsabilités que j'ai au PSG mais mes dirigeants, eux, ne les voient pas $»^{11}$. Dans les deux cas, et compte tenu des difficultés à recruter un substitut de qualité à leur poste respectif, la stratégie développée par les joueurs a fonctionné, menant à la réalisation effective du hold-up. Chacun d'entre eux a obtenu une prolongation de contrat assortie d'une revalorisation salariale, d'un montant annuel de 5 millions d'euros pour S. Ramos (son salaire annuel passant de 5 à 10 millions d'euros) ${ }^{12}$ et d'environ 1,5 million d'euros pour T. Motta (son salaire passant de 6,3 à 7,8 millions d'euros par an) ${ }^{13}$.

Concernant la seconde hypothèse, les illustrations sont également nombreuses. À titre d'exemple, les performances sportives de Morgan Schneiderlin et de Clinton Njie, respectivement à Southampton et au sein de l'olympique Lyonnais, leur ont permis de rejoindre en 2015 Manchester United pour le premier et Tottenham pour le second. M. Schneiderlin a alors doublé son salaire annuel (de 4 à 8 millions d'euros), celui de C. Njie étant passé de 360000 euros à 2,2 millions d'euros. Plus récemment, la rémunération salariale de Gonzalo Higuain a progressé de 2 millions d'euros (de 5,5 à 7,5 millions d'euros annuels) lors de son transfert du SSC Napoli à la Juventus de Turin en 2016.

Dans les faits, il est courant qu'un résultat hybride survienne : club et joueur s'accordent d'abord sur une prolongation contractuelle assortie d'une revalorisation salariale ; dès la saison suivante, le joueur quitte le club pour un autre. Cette solution permet au club de pérenniser ses performances sportives et financières, d'une part, et d'ajuster sa politique sportive et de recrutement au départ du joueur, d'autre part. L'exemple de Grzegorz Krychowiak et du Stade de Reims en est représentatif. À l'issue de la saison 2012/2013 de Ligue 1, le club est parvenu à conserver le joueur une année supplémentaire au moyen d'une revalorisation salariale, alors que l'international polonais souhaitait quitter le club rémois afin de capitaliser sur ses bonnes performances sportives. Dès la saison 2014/2015, G. Krychowiak a rejoint le club espagnol du FC Séville qui lui proposait un salaire de près de 1 million d'euros par an contre les 400000 euros annuels qu'il percevait au Stade de Reims. Quoi qu'il en soit, d'un point de vue théorique, ce résultat confirme la présence 
d'une situation de hold-up dans la mesure où le joueur assoit un pouvoir de négociation qui lui permet de capter des revenus supplémentaires.

Conformément au problème du hold-up et à ses deux issues, il n'est pas étonnant de constater que les joueurs captent une large partie des revenus dégagés par les clubs. Ainsi, depuis le début des années 2000, les clubs issus des ligues du Big Five consacrent plus de la moitié de leurs recettes aux salaires des joueurs (graphique 1). Hormis le cas allemand, d'un côté, où les clubs maintiennent cette part à $51 \%$ en moyenne sur la période 2000-2014, et à l'exception du cas italien, de l'autre, où les clubs consacrent en moyenne $71 \%$ de leurs revenus aux salaires sur la même période, la moyenne se situe plutôt entre $60 \%$ et $70 \%$ dans les trois autres ligues : $63 \%$ en Liga espagnole, $64 \%$ en Premier League anglaise et $67 \%$ en Ligue 1 française.

Graphique 1. Salaires des joueurs dans les cinq ligues nationales majeures en Europe (Big Five), en pourcentage des revenus des clubs

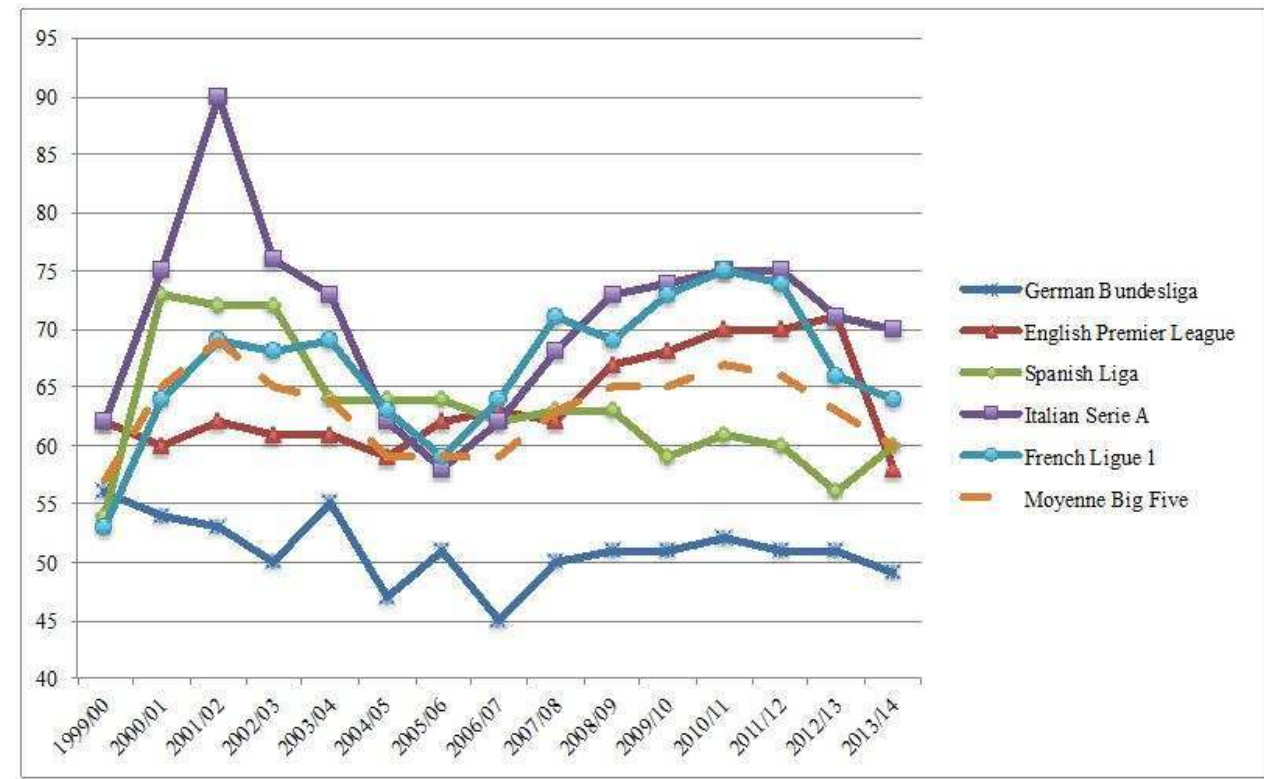

Source : auteur, d'après Andreff, Deloitte, DNCG et UEFA

En élargissant à l'ensemble des premières divisions européennes, ce ratio salaires/ revenus s'est élevé à $62 \%$ en 2016, pourcentage moyen du chiffre d'affaires affecté par les clubs européens à la masse salariale depuis 1997. Dans ce contexte, de nombreux clubs consacrent plus de $70 \%$ voire l'intégralité de leurs revenus aux salaires. À l'échelle européenne, et à partir des données disponibles (tableau 1), 259 clubs ont affecté plus de $70 \%$ de leurs recettes à la masse salariale en 2014. 271 clubs étaient concernés en 2012, parmi lesquels 94 ont enregistré des charges salariales supérieures à leurs recettes totales. Sur ce dernier point, et à titre d'illustration, durant la saison 2011/2012, deux clubs de Ligue 1 ont enregistré une masse salariale supérieure à leurs revenus (DNCG), à savoir le Lille Olympique Sporting Club (109\%) et l'AJ Auxerre (116\%). L'ensemble de ces données démontre que les joueurs sont capables de s'approprier une large partie des revenus générés par le football professionnel, à commencer par les droits télévisés . 
Tableau 1. Ratio salaires/revenus et nombre de clubs affectant plus de $70 \%-100 \%$ de leurs revenus aux salaires, ensemble des $1^{\text {res }}$ divisions européennes

\begin{tabular}{|c|c|c|c|}
\cline { 3 - 4 } \multicolumn{1}{c|}{} & \multirow{2}{*}{ Sal ai res Revenus } & \multicolumn{2}{c|}{ Nombre de clubs avec un ratio } \\
\cline { 3 - 4 } \multicolumn{1}{c|}{} & & $\mathbf{> 7 0} \%$ & $\mathbf{> 1 0 0 \%}$ \\
\hline $\mathbf{2 0 0 8}$ & 62 & 198 & 57 \\
\hline $\mathbf{2 0 0 9}$ & 64 & 249 & 73 \\
\hline $\mathbf{2 0 1 0}$ & 64 & 254 & 78 \\
\hline $\mathbf{2 0 1 1}$ & 65 & 257 & 88 \\
\hline $\mathbf{2 0 1 2}$ & 65 & 271 & 94 \\
\hline $\mathbf{2 0 1 3}$ & 64 & 270 & $n d$ \\
\hline $\mathbf{2 0 1 4}$ & 62 & 259 & $n d$ \\
\hline $\mathbf{2 0 1 5}$ & 63 & $n d$ & $n d$ \\
\hline $\mathbf{2 0 1 6}$ & 62 & $n d$ & $n d$ \\
\hline
\end{tabular}

Source : auteur, d'après UEFA

De plus, étant donné que les revenus des clubs de première division ne cessent de croître depuis de nombreuses années, les salaires des joueurs progressent eux-aussi. Plus encore, sur longue période, la croissance des salaires a été plus rapide que la croissance des revenus des clubs - ce que montrent les données agrégées au niveau européen (graphique 2) ${ }^{14}$ et l'exemple français (graphique 3) ${ }^{15}$ - augmentant de fait le ratio salaires/revenus dans plusieurs ligues nationales. Par conséquent, le problème du hold-up constitue un facteur explicatif supplémentaire à l'inflation salariale dans le football professionnel en Europe. Bien qu'il existe des irrégularités se caractérisant par des départs de joueurs dans d'autres clubs sans augmentation de salaire, la tendance va à la revalorisation salariale des joueurs à chaque renouvellement ou à chaque nouveau contrat ${ }^{16}$.

Graphique 2. Évolution comparée de la croissance de la masse salariale et des revenus des clubs européens de $1^{\text {re }}$ division (en millions d'euros)

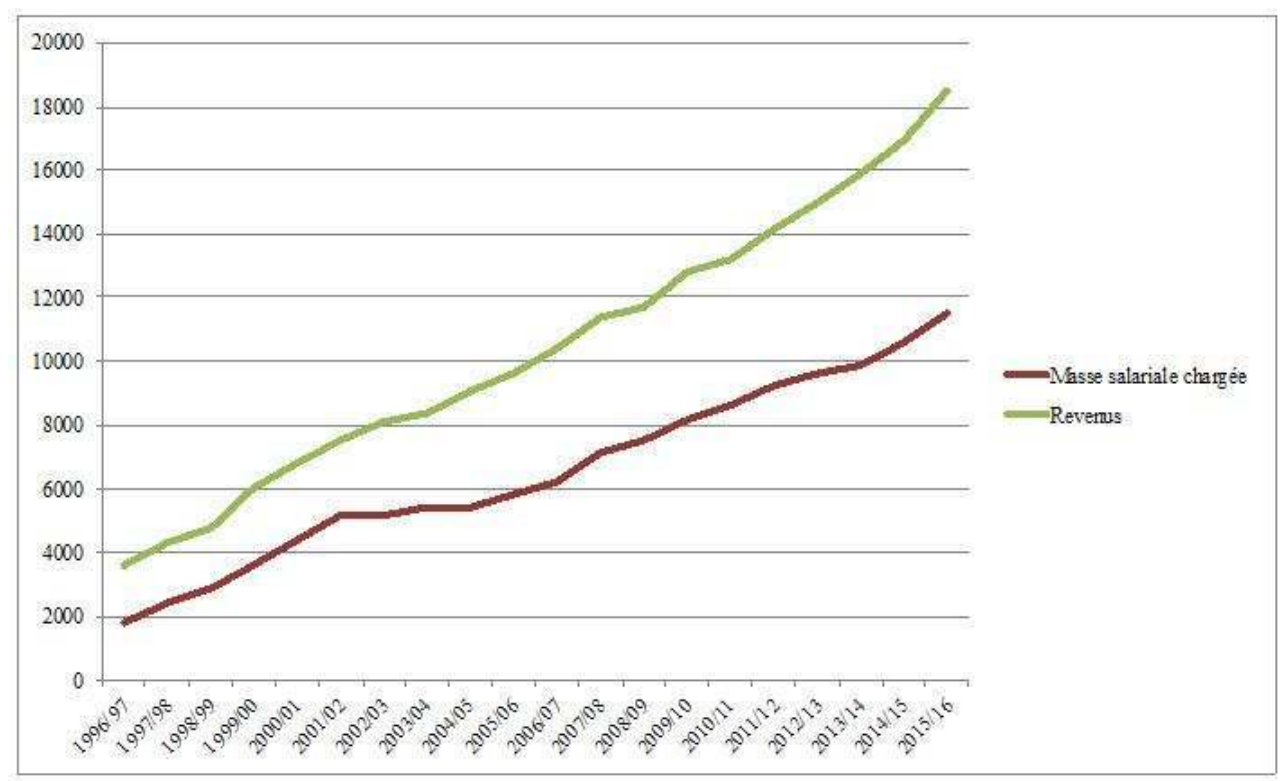

Source : auteur, d'après UEFA 
Graphique 3. Évolution comparée de la croissance de la masse salariale et des revenus des clubs français de $1^{\text {re }}$ division (en millions d'euros)

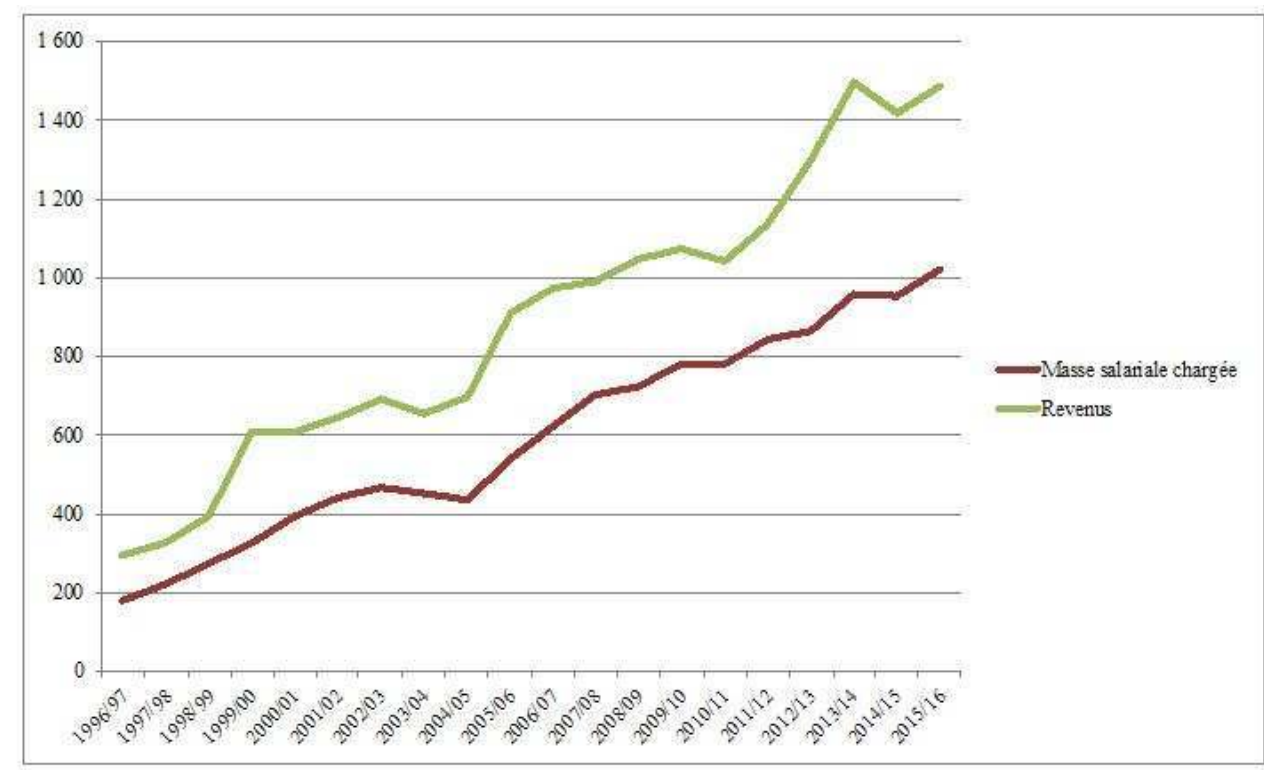

Source : auteur, d'après DNCG

\section{Les réactions stratégiques des clubs au problème du hold-up}

\subsection{De l'allongement de la durée des contrats à l'augmentation du prix des transferts}

Dès l'instant où ils sont confrontés à une stratégie de retrait développée par un de leurs joueurs, les clubs réalisent un calcul basé sur les pertes que pourraient engendrer le départ du joueur (par sa triple valeur) par rapport à la revalorisation salariale qu'ils seraient prêt à lui accorder pour le retenir. Ces estimations mènent un club à deux alternatives :

a. si les pertes anticipées sont inférieures au salaire que le club est prêt à consentir pour conserver le joueur (pertes < salaire), alors le club décide de ne pas retenir le joueur.

b. si les pertes anticipées sont supérieures au salaire que le club est prêt à consentir pour préserver le joueur (pertes > salaire), alors le club décide de conserver le joueur.

41 Ce choix est complexe pour les clubs étant donné qu'il repose sur des prévisions (relatives à la valeur des joueurs) qui ne peuvent être vérifiées qu'au terme du contrat - voire à chaque fin de saison sportive. Ce n'est qu'à ce moment que les clubs sont en mesure de déterminer si les revenus engendrés par un joueur par ses valeurs d'usage et de nonusage compensent leurs dépenses salariales, i.e. si leurs anticipations étaient bonnes.

Cette difficulté se pose également pour évaluer la valeur des substituts potentiels au joueur menaçant de se retirer, ce qui complexifie davantage encore le calcul réalisé par les clubs soumis au risque de hold-up. Dans cette perspective, l'arbitrage réalisé par les clubs doit plus largement considérer le problème de rareté, en ce sens qu'ils doivent intégrer à leurs calculs l'état actuel du marché du travail, i.e. les éventuelles difficultés de 
recrutement. Les clubs doivent être en mesure d'identifier si des substituts de qualité sont disponibles et accessibles à l'instant $t$. Ainsi, tout recrutement peut s'avérer ex post comme constitutif d'un actif spécifique dès lors qu'un club connaît des difficultés de remplacement d'un joueur en partance. La rareté est plus intense pour certains postes de jeu, à commencer par les attaquants. Par conséquent, ces joueurs disposent d'un pouvoir de négociation supérieur qui leur permet de percevoir des salaires plus élevés . Le pouvoir de négociation des joueurs est également influencé par leur âge et par leur expérience ( ibid.). Les clubs supportent donc des coûts de transaction liés à la recherche de nouveaux joueurs et aux négociations qui en découlent. De même, le recrutement de nouveaux joueurs peut être à l'origine de coûts d'insertion . Enfin, les clubs doivent intégrer à leurs calculs les potentiels clubs acheteurs du joueur menaçant d'un départ. Il s'agit de prendre en compte l'éventuel renforcement de la concurrence selon le club de destination du joueur. En résumé, les clubs doivent nécessairement incorporer à leurs anticipations le coût global de remplacement d'un joueur (ibid.).

43 Finalement, quelle que soit l'alternative choisie, un club est nécessairement affecté par une stratégie de retrait. S'il décide de se séparer effectivement du joueur menaçant de se retirer (a), il met fin à un actif cospécialisé dont il s'appropriait une partie de la rente (et dans lequel il a investi). Dans le cas contraire, s'il décide et parvient à retenir le joueur (b), le club continue de bénéficier de la rente grâce au maintien de leur relation contractuelle, mais il est dépossédé d'une fraction supplémentaire du surplus qu'ils dégagent collectivement puisqu'il la consacre à la revalorisation salariale du joueur. C'est pourquoi les clubs cherchent à se prémunir de la situation de hold-up et de ses conséquences. Pour cela, leur objectif est d'abord de contenir les stratégies opportunistes post-contractuelles des joueurs en limitant les périodes de renégociation. L'allongement de la durée des contrats est l'instrument principalement utilisé par les clubs afin de satisfaire cet objectif. Il s'agit d'une des deux solutions précontractuelles que soulignent les études empiriques sur le problème du hold-up. Cette pratique est d'autant plus adoptée par les clubs qu'ils consentent à d'importants investissements spécifiques dans les joueurs . Cela étant, en vertu du Monti system de 2001, la durée des contrats est limitée à cinq ans. Par ailleurs, conformément à cette réglementation, les joueurs peuvent quitter un club pour un autre avant la fin de leur contrat en échange d'une indemnité de transfert . Autrement dit, un joueur sous contrat peut être transféré d'un club à un autre à condition que le club acheteur verse une indemnité au club vendeur. Il y a acte d'achat de contrat ${ }^{17}$.

Dans ce contexte, la réaction stratégique majeure des clubs au problème du hold-up est de gonfler le prix des joueurs lors des transferts. Le but est d'obtenir une indemnité élevée qui va compenser les pertes liées au départ des joueurs, c'est-à-dire une indemnité qui va internaliser les coûts que génère le hold-up. En vertu des dispositions de l'arrêt Bosman, cette stratégie implique que les joueurs ne soient pas en fin de contrat car, dans ce cas, aucune indemnité de transfert n'est versée. Par conséquent, au-delà du fait de limiter les périodes de renégociation, l'allongement de la durée des contrats est aussi un instrument qui permet aux clubs de maintenir un joueur sous contrat. Dès lors, tout départ engendre le versement d'une indemnité de transfert. Dans un objectif identique, une pratique courante des clubs est de renouveler régulièrement les contrats de joueurs avant leur terme, et plus précisément avant la dernière année de contrat. Cette prolongation contractuelle est néanmoins assortie d'une revalorisation salariale. À nouveau, les exemples sont nombreux. Prenons celui de l'international belge Eden Hazard. Recruté en 2012 par Chelsea, son contrat a été prolongé pour trois saisons supplémentaires en 2015, 
le joueur étant dorénavant lié au club jusqu'en 2020. Dans le cadre de cette prolongation contractuelle, E. Hazard a vu son salaire passer de 8,5 millions d'euros annuels à 13 millions d'euros par an. En définitive, depuis l'arrêt Bosman, si aboutissent les stratégies d'allongement de la durée des contrats poursuivies par les clubs, c'est parce que les joueurs y trouvent leur intérêt. Cet allongement contractuel engendre une hausse des salaires individuels des joueurs (Frick 2007; Tang 2015), même si ceux du segment supérieur du marché du travail augmentent plus vite que ceux du segment inférieur, creusant les inégalités salariales .

Afin de déterminer le montant minimum de l'indemnité pour laquelle ils sont prêts à «vendre " un joueur, les clubs prennent en compte la productivité du joueur. En pratique, puisque celle-ci est complexe à déterminer ${ }^{18}$, les clubs vendeurs estiment la productivité de leur joueur par la somme des salaires restant dus jusqu'à la fin du contrat, ce qui leur permet de fixer un montant minimum pour le transfert (Brocard 2010). L'implication est double : plus il reste d'années avant le terme du contrat, plus le prix du transfert sera élevé (Feess et al., 2015; Frick 2007) alors même que la probabilité que le transfert soit réalisé diminue ; plus le salaire est élevé, plus l'indemnité de transfert sera importante. Pour s'assurer du versement d'une indemnité de transfert minimum, certains clubs instaurent des clauses libératoires au contrat de leurs joueurs. Elles indiquent la somme minimum qu'un club doit acquitter pour obtenir un joueur sous contrat. À titre d'exemple, le SSC Napoli avait assorti le contrat d'E. Cavani d'une clause libératoire de 64 millions d'euros, somme versée par le PSG en 2013 pour acquérir l'attaquant uruguayen. Le club italien a alors pu engager un substitut de qualité en la personne de G. Higuain qui évoluait au Real Madrid pour 40 millions d'euros. Il est à noter que les clauses libératoires sont souvent surévaluées, l'objectif sous-jacent des clubs étant de décourager la concurrence dans l'obtention du joueur. La clause libératoire incorporée au contrat de C. Ronaldo par le Real Madrid s'élève par exemple à 1 milliard d'euros. Le transfert de Neymar au PSG en août 2017 a néanmoins remis en cause le niveau à partir duquel ces clauses sont opérantes: le club a en effet payé la clause libératoire de 222 millions d'euros prévue par le contrat qui liait le joueur brésilien au FC Barcelone afin de le recruter ${ }^{19}$.

Du côté du club acheteur, celui-ci cherche à prévoir ce que le joueur qu'il désire recruter peut lui apporter, à la fois par sa valeur d'usage et sa valeur de non-usage. Il tente alors d'évaluer si les revenus espérés par le recrutement du joueur peuvent compenser, voire excéder, l'indemnité de transfert à payer augmentée des salaires à verser durant le contrat. L'objectif du club est d'obtenir un retour sur investissements .

\subsection{L'accroissement des indemnités de transfert : d'une réponse stratégique privilégiée au hold-up à un facteur de déséquilibre des clubs}

Cette stratégie des clubs de prise en charge des effets négatifs du hold-up par les transferts est marquée par son effet boule de neige. Force est de constater que la valeur des transferts est en constante augmentation. D'un côté, le montant minimum des indemnités de transfert demandé par les clubs vendeurs s'accroît au rythme de l'inflation des salaires des joueurs et, de l'autre, les clubs acheteurs surestiment continuellement la valeur des 
joueurs et proposent des indemnités élevées assorties de hauts salaires. Trois raisons peuvent expliquer cette surévaluation des clubs acheteurs :

i. En situation d'asymétrie d'information, les clubs forment des anticipations optimistes ou erronées - dans lesquelles les agents de joueurs peuvent jouer un rôle - qui augmentent mécaniquement la valeur des joueurs.

ii. Les clubs qui bénéficient d'un "dopage financier", sous quelque forme que ce soit, n'attendent pas un retour sur investissement direct lors d'un recrutement. Ils sont instrumentalisés à des fins extrasportives : objectif stratégique de long terme, création de synergies sectorielles, diffusion d'une image positive ou encore blanchiment d'argent .

iii. La concurrence entre clubs pèse à la hausse sur le niveau des indemnités de transfert.

Même si le premier facteur ne peut être négligé (i), la conjugaison des deux autres (ii et iii) offre une explication fiable à la hausse des prix des transferts. Le mécanisme explicatif rejoint d'ailleurs celui permettant de restituer l'inflation salariale dans le football au regard des répercussions des effets inflationnistes du segment primaire sur le segment secondaire du marché du travail . Ainsi, les clubs soutenus par des bailleurs à fonds perdus sont en mesure de proposer des indemnités de transfert élevées pour certains types de joueurs (i.e. les superstars), ce qui résulte de la concurrence que se livrent ces clubs sur le segment primaire du marché du travail (processus de surenchérissement). Parce qu'ils doivent rester compétitifs (ligue ouverte), les clubs qui disposent d'une manne financière plus restreinte se font concurrence sur le segment secondaire du marché du travail afin d'obtenir les meilleurs joueurs possibles. Ce processus conduit lui-aussi à une augmentation des prix des transferts sur ce segment du marché sous l'impulsion des revenus issus des droits télévisés. D'autant plus que les "grands » clubs sont eux aussi actifs sur ce segment du marché. Ils y ont recours afin de recruter des joueurs pour lesquels il n'existe pas ou peu de substituts disponibles à ce poste sur le segment supérieur, soit afin de compléter leur vaste effectif. Au final, les clubs réalisent des investissements dont la rentabilité est limitée, et qui contribuent à l'inflation du prix des transferts sur l'ensemble du marché.

Les dépenses de transferts des clubs européens ont donc fortement augmenté à partir des années 1970, avec un net accroissement à la fin des années 1990 sous l'effet de l'arrêt Bosman. L'augmentation de la durée des contrats n'a finalement pas affecté la mobilité des joueurs (Frick 2007; Frick \& Simmons 2014). Au contraire, cette dernière s'est intensifiée, entraînant avec elle une augmentation continue des prix des transferts (tableau 2). Par exemple, au sein du Big Five, la croissance des dépenses de transferts a été de 950 \% entre les saisons 1994/1995 et 2014/2015. À l'échelle de l'ensemble des premières divisions européennes, les dépenses de transferts brutes des clubs ont dépassé 5,4 milliards d'euros sur l'exercice 2015 (UEFA). 
Tableau 2. Balance des transferts des $1^{\text {res }}$ divisions anglaise (Premier League) et française (Ligue 1), et dépenses de transferts cumulées des cinq ligues nationales majeures en Europe (Big Five), en millions d'euros

\begin{tabular}{|c|c|c|c|c|c|c|c|}
\cline { 2 - 8 } \multicolumn{1}{c|}{} & \multicolumn{3}{c|}{ PL } & \multicolumn{3}{c|}{ L1 } & BigFive \\
\cline { 2 - 8 } \multicolumn{1}{c|}{ Dépenses } & Recettes & Résultat & Dépenses & Recettes & Résultat & Dépenses \\
\hline $\mathbf{2 0 0 9 / 1 0}$ & 1210 & 688 & -522 & 171 & 228 & 57 & 2719 \\
\hline $\mathbf{2 0 0 4 / 0 5}$ & 501 & 183 & -318 & 141 & 225 & 84 & 1159 \\
\hline $\mathbf{1 9 9 9 / 0 0}$ & 312 & 226 & -86 & 106 & 86 & -20 & 1215 \\
\hline $\mathbf{1 9 9 4 / 9 5}$ & 137 & 92 & -45 & 0 & 3 & 3 & 259 \\
\hline $\mathbf{1 9 8 9 / 9 0}$ & 36 & 28 & -8 & 8 & 4 & -4 & 89 \\
\hline $\mathbf{1 9 8 4 / 8 5}$ & 10 & 9 & -1 & - & - & - & 37 \\
\hline $\mathbf{1 9 7 9 / 8 0}$ & 12 & 10 & -2 & - & - & - & 20 \\
\hline
\end{tabular}

Source : auteur, d'après transfertmarkt.fr fait, si le pourcentage de transferts payants n'a pas augmenté après l'arrêt Bosman en raison de la suppression de la compensation financière de fin de contrat (Brocard 2015; Frick 2007), les indemnités de transfert ont quant à elles augmenté en se concentrant sur un nombre limité de joueurs - i.e. ceux qui composent le segment haut du marché du travail pour qui les indemnités se sont nettement accrues - ce que confirme le récent transfert de Neymar. Si cette mutation marque une nette hausse de la valeur maximum attribuée à un footballeur professionnel, elle ne constitue pas une rupture au sens où elle s'inscrit dans une tendance longue d'inflation du prix des transferts, la valeur des meilleurs joueurs dépassant aujourd'hui les 100 millions d'euros: Ousmane Dembélé et Paul Pogba respectivement acquis pour 105 millions d'euros par le FC Barcelone en 2017 et par Manchester United en 2015, ou encore Gareth Bale acheté par le Real Madrid en 2013 contre 101 millions d'euros.

51 En outre, bien que les transferts sans indemnités aient augmenté, les primes à la signature se sont développées en parallèle . Elles concernent aussi bien les transferts avec ou sans indemnités. À titre d'exemple, d'un côté, C. Ronaldo a coûté 10 millions d'euros de prime à la signature en supplément aux 94 millions d'euros déboursés par le Real Madrid pour le recruter en 2009; de l'autre, André Ayew a reçu une prime à la signature d'environ 8 millions d'euros lorsqu'il a quitté gratuitement l'OM en 2015 au terme de son contrat pour rejoindre le club anglais de Swansea.

À ces primes de signature, s'ajoutent une multitude de primes sous forme de clauses conditionnelles qui complexifient les contrats de joueurs tout en augmentant leur rémunération salariale: primes d'apparition, de performances, de qualification, de résultats, etc. . Selon nous, ce développement de la partie variable de la rémunération des joueurs peut se comprendre à la lumière du problème du hold-up. Après la signature d'un 
contrat, en raison d'une situation d'aléa moral, les joueurs peuvent être incités à limiter leurs performances sportives dans la mesure où ils sont " couverts " par un contrat de longue durée (Feess \& Muehlheusser 2003 ; Frick 2007). Avant la fin de leur contrat, les joueurs cherchent, en règle générale, à rétablir de bonnes performances sportives afin d'augmenter leur valeur marchande. C'est donc dans l'optique de contrer cet opportunisme post-contractuel des joueurs que les clubs développent l'utilisation des primes.

Ce développement progressif du niveau des transferts et des primes en réaction au risque $\mathrm{du}$ hold-up est néanmoins problématique dès lors que l'opération implique un club étranger. Plus précisément, lorsqu'un club européen achète un joueur à un club noneuropéen, la transaction s'accompagne d'un flux de trésorerie du club acheteur au club vendeur qui entraîne une fuite de liquidité hors du circuit continental fragilisant la situation financière des clubs européens. Ainsi, le déficit de la balance des transferts des ligues du Big Five (650 millions d'euros) durant la saison 2014/2015, largement dû au solde négatif de la balance des transferts de la Premier League (522 millions d'euros), peut à la fois s'interpréter comme une détérioration financière globale du football européen dans les cas où ce déficit a financé l'achat de joueurs issus de clubs étrangers (transferts extraeuropéens), et comme un simple transfert d'argent des ligues du Big Five aux autres ligues européennes dans les cas où ce déficit a financé l'achat de joueurs provenant d'autres clubs localisés en Europe (transferts intra-européens).

Pour autant, il ne peut pas être avancé que le développement excessif des transferts entre clubs européens est vertueux. Ils comportent selon nous deux effets néfastes. Le premier tient au fait qu'une proportion des gains réalisés sur les ventes de joueurs est réaffectée aux dépenses salariales des clubs, et contribue donc à alimenter l'inflation salariale. Le second repose sur l'existence de dettes de transfert. Elles caractérisent des impayés entre clubs relatifs aux activités de transfert, ce qui signifie qu'un club n'a pas réglé l'intégralité de l'indemnité due à un autre club dans le cadre d'une mutation de joueur. Le club vendeur se voit alors à la fois dépourvu de son joueur et privé des sommes liées à la transaction. Cet endettement s'élevait à 2,4 milliards d'euros en 2012 (UEFA).

L'évolution du marché mondial des transferts, qui se caractérise par l'inflation des salaires et des prix des joueurs, a donc participé à la fragilisation de la situation financière des clubs européens. Les soldes négatifs des transferts, les amortissements des contrats de joueurs et les salaires versés (primes comprises) alourdissent les charges financières des clubs et creusent leurs déficits. À ce titre, depuis 2008, le résultat net cumulé des clubs européens de première division est constamment négatif, plus de la moitié d'entre eux présentant régulièrement un exercice déficitaire (UEFA). De 2009 à 2012, leur déficit net s'est élevé à plus de 1 milliard d'euros et il a même été supérieur à 1,5 milliard d'euros en 2010 et en 2011, avant de réduire progressivement les saisons suivantes sous l'effet de l'instauration du fair-play financier en 2012 (Bastien 2017). Le déficit net des clubs est ainsi passé de 767 millions d'euros en 2013 à 269 millions d'euros en 2016 (UEFA). Le recours à l'endettement permet de financer ces déficits récurrents et il permet à terme de répondre indirectement aux dépenses salariales et de transferts des clubs. Entre 2009 et 2016, les dettes nettes des clubs européens de première division se sont élevées à plus de 6,9 milliards d'euros en moyenne, celles-ci étant néanmoins passées de 7,6 milliards d'euros à 6,4 milliards d'euros sur la période (ibid. ${ }^{20}$. En définitive, une pratique dont l'objectif originel est de limiter les conséquences du problème du hold-up 
contribue paradoxalement à fragiliser davantage la situation financière des clubs. Les réactions stratégiques des clubs au hold-up engendrent peu d'effets compensatoires.

\section{Conclusion} propres à la relation salariale dans le football professionnel européen. Parce que leur comportement post-contractuel basé sur des stratégies de retrait réel ou fictif leur permet généralement de percevoir une rémunération plus élevée, les joueurs se révèlent être les acteurs influents du football. Ils captent les richesses du secteur par les deux modalités relatives au problème du hold-up : soit par l'obtention d'une revalorisation salariale dans leur club (réalisation effective du hold-up), soit par l'acquisition d'un meilleur salaire dans un autre club (nouvelle relation contractuelle). À ce titre, nous faisons l'hypothèse, dans ce travail, que le problème du hold-up n'est pas restreint au marché primaire du travail et qu'il peut s'étendre au marché secondaire. À l'égard de la segmentation du marché du travail, la seule différence tient au fait que les superstars sont capables d'instaurer un rapport de force plus intense et plus ample lié à leur faible substituabilité, et qui se traduit par un prélèvement de revenus plus important. En conséquence, le processus de hold-up n'évacue en rien l'existence de fortes inégalités dans le football professionnel. Plus encore, les faibles rémunérations perçues et la précarité subie par une fraction des joueurs du marché secondaire s'expliquent par leur faible pouvoir de négociation face aux clubs. Le positionnement des joueurs dans leurs rapports aux clubs (favorable ou défavorable) est donc fonction de leur degré de spécificité et de leur contribution au surplus dégagé collectivement.

part, le problème du hold-up permet d'éclairer les stratégies développées par les clubs, ces derniers cherchant en fait à limiter les conséquences du hold-up qui peuvent leur être néfastes. Au-delà de l'allongement de la durée des contrats, l'apport de notre travail réside surtout en la reconnaissance de la hausse des indemnités de transfert comme un résultat des réactions stratégiques des clubs au problème du hold-up, ce qui explique leur niveau actuel. Cependant, la croissance continue des prix des transferts aboutit à un effet inverse : l'accroissement des déséquilibres financiers des clubs, surtout lorsqu'il s'agit d'achats de joueurs qui n'évoluent pas en Europe. Ainsi, au contraire de certains secteurs ou de certaines industries, comme la finance, dans lesquels les coûts salariaux sont les coûts les plus élevés pour les employeurs, ce qui se traduit par l'octroi de rémunérations élevées aux salariés, les hauts salaires perçus par les footballeurs professionnels qui évoluent en Europe ne s'expliquent aucunement par la rentabilité des entreprises qui les emploient. En effet, ces dernières ne sont pas (ou peu) rentables pour la plupart.

59 L'application au football du problème du hold-up proposée dans cet article appelle à un prolongement évident. Ce sport, comme tous les sports professionnels (principalement collectifs), repose sur une spécificité tenant à l'existence d'un intermédiaire sur le marché 
du travail (Brocard 2010). Par conséquent, les relations contractuelles entre les clubs et les joueurs, sans oublier les jeux de négociation qu'elles impliquent, ne peuvent être étudiées sans s'interroger plus en profondeur sur le rôle qu'y jouent les agents sportifs. Ces derniers étant principalement mandatés par les joueurs, la question de leur influence dans le processus de formation du pouvoir de négociation des footballeurs se pose plus particulièrement.

\section{BIBLIOGRAPHIE}

Aglietta M., Andreff W. \& B. Drut (2008), « Bourse et football », Revue d'Économie Politique, vol. 118, $\mathrm{n}^{\circ} 2$, p. 255-292.

Alchian A. A. \& H Demsetz (1972), « Production, Information Costs, and Economic Organization », American Economic Review, vol. 62, n 5, December, p. 777-795.

Andreff W. (2007), « Régulation et institutions en économie du sport », Revue de la régulation [En ligne], 1 | Juin / June 2007, mis en ligne le 25 juin 2007, consulté le 31 mai 2018. URL : http:// journals.openedition.org/regulation/1274 ; DOI : 10.4000/regulation.1274

Andreff W. (2009), «Équilibre compétitif et contrainte budgétaire dans une ligue de sport professionnel », Revue économique, volume 60, n³ 3, p. 591-633.

Andreff W. (2014), « French professional football: how much different? », in Goddard J. \& P. Sloane, Handbook on the Economics of Professional Football, Cheltenham, Northampton, Edward Elgar, p. 298-321.

Andreff W. \& P. D. Staudohar, (2000), « The evolving European model of professional sports finance ", Journal of Sports Economics, vol. 1, n 3, p. 257-276.

Aoki M. (2006), Fondements d'une analyse institutionnelle comparée, Paris, Albin Michel.

Bastien J. (2017), « Le football professionnel en Europe est-il en crise ? Une réponse régulationniste », Revue de la régulation [En ligne], 21 | 1er semestre / Spring 2017, mis en ligne le 04 juillet 2017, consulté le 31 mai 2018. URL : http://journals.openedition.org/ regulation/12282; DOI : 10.4000/regulation.12282

Bourg J.-F. (1983), « Salaire, travail et emploi dans le football professionnel français », Paris, Fédération Française de Football, Ligue Nationale de Football.

Bourg J.-F. (1988), « L'inflation salariale », Problèmes politiques et sociaux, vol. 581, p. 52-53.

Bourg J.-F. (1990), « Le marché du travail des footballeurs : dualisme et rapport salarial », Problèmes économiques, vol. 2174.

Bourg J.-F. (1998), « Dualisme et rapport salarial dans le sport professionnel », in Bourg J.-F. \& J.J. Gouguet, Analyse économique du sport, Paris, Puf, p. 119-179.

Bourg J.-F. (2008), « Les très hauts revenus des superstars du sport : un état des approches théoriques et empiriques ", Revue d'économie politique, vol. 118, n 3, p. 375-394 ; doi:10.3917/ redp.183.0375 
Bouvet P. (1996), «Les salaires des "vedettes" du sport professionel par équipe : une application privilégiée de la théorie du salaire d'efficience? », Revue française d'économie, vol. 11, n 4 , p. 119-144.

Bouvet P. \& C. Lepetit (2015), « Du dopage au dopage financier », Reflets \& Perspectives de la vie économique, $\mathrm{n}^{\circ}$ 3, tome LIV, p. 39-56.

Brocard J.-F. (2010), « Marché des transferts et agents sportifs : le dessous des cartes », Géoéconomie, $\mathrm{n}^{\circ}$ 54, p. 79-89.

Brocard J.-F. (2015), « Transferts de joueurs et "Third party ownership" ", Reflets \& Perspectives de la vie économique, $\mathrm{n}^{\circ} 3$, tome LIV, p. 57-69.

Coase R. (1937), « The nature of the firm », Economica, New Series, vol. 4, n 16, p. 386-405.

Coriat B. \& O. Weinstein (2010), « Les théories de la firme entre "contrats” et "compétences". Une revue critique des développements contemporains ", Revue d'économie industrielle, volume 129-130, n 1, p. 57-86.

Deloitte (2008-2017), Annual Review of Football Finance, éditions 2008 à 2017.

DNCG (2002-2017), Rapports de contrôle des clubs professionnels. Situation du football professionnel, Direction nationale du contrôle de gestion, saisons 2000/2001-2015/2016.

Doeringer P. \& M. Piore (1971), Internal Labour Markets and Manpower Analysis, Massachusetts, Lexington Books.

Dubrion B. (2002), «Allocation et incitation intra-firme : d'une évaluation de l'approche contractualiste à l'élaboration d'un cadre d'analyse des "dispositifs de gestion de la relation d'emploi". Application au cas d'une grande banque régionale », Thèse sous la direction de B. Baudry, Université Lumière Lyon 2.

Feess E., Gerfin M. \& G. Muehlheusser (2015), « Contracts as rent-seeking devices: evidence from german soccer », Economic Inquiry, vol. 53, n 1, p. 714-730.

Feess E. \& G. Muehlheusser (2003), « The impact of transfer fees on professional sports: an analysis of the new transfer system for european football ", The Scandinavian Journal of Economics, vol. $105, \mathrm{n}^{\circ} 1$, p. 139-154.

Frick B. (2007), « The football players' labor market: empirical evidence from the major european leagues », Scottish Journal of Political Economy, vol. 54, n 3, p. 422-446.

Frick B. (2011), « Performance, salaries and contract lenght: empirical evidence from german soccer », International Journal of Sport Finance, vol. 6, $\mathrm{n}^{\circ}$ 2, p. 87-118.

Frick B.\& R. Simmons (2014), « The footballers' labour market after the Bosman ruling », in Goddard J. \& P. Sloane, Handbook on the Economics of Professional Football, Cheltenham, Northampton, Edward Elgar, p. 203-226.

Gerrard B. (2006), «Financial innovation in professional team sports: the case of English Premiership soccer ", in Andreff W. \& S. Szymanski, Handbook on the Economics of Sport, Cheltenham, Edward Elgar, p. 709-718.

Godechot O. (2006), « Hold-up en finance. Les conditions de possibilité des bonus élevés dans l'industrie financière », Revue française de sociologie, vol. 47, nº 2, p. 341-371.

Gouguet J.-J. \& D. Primault (2004), « Analyse économique du fonctionnement du marché des transferts dans le football professionnel ", in Gouguet J.-J., Le sport professionnel après l'arrêt Bosman : une analyse économique internationale, Limoges, Pulim, p. 113-142. 
Grossman S. \& O. Hart (1986), « The Costs and Benefits of Ownership: A Theory of Vertical and Lateral Integration », Journal of Political Economy, vol. 94, n 2, p. 691-719.

Hart O. (1995), Firms, Contracts, and Financial Structure, New-York, Oxford University Press. Jensen M. \& W. Meckling, (1976), « Theory of the Firm: Managerial Behavior, Agency Costs and Ownership Structure ", Journal of Financial Economics, vol. 3, n 4, p. 305-360.

Joskow P. L. (1985), « Vertical integration and long-term contracts: the case of coal-burning electric generating plants ", Journal of law, economics \& organization, volume 1, n 1, p. 33-80.

Kesenne S. (1996), «League management in professional team sports within win maximizing clubs », European journal of sport management, $\mathrm{n}^{\circ} 2$.

Klein B., Crawford R. G. \& A. A. Alchian (1978), « Vertical integration, appropriable rents, and the competitive contracting process ", Journal of law and economics, volume 21, n² 2, p. 297-326.

Lazear E. P. \& S. Rosen (1981), « Rank-order tournaments as optimum labor contracts », Journal of Political Economy, volume 89, p. 841-864.

Lyon T. P. \& E. Rasmusen (2004), « Buyer-Option Contracts Restored: Renegotiation, Inefficient Threats, and the Hold-Up Problem », The Journal of Law, Economics, and Organization, vol. 20, n 1 , p. $148-169$.

Malcomson J. M. (1997), « Contracts, Hold-up, and Labor Markets », Journal of Economic Literature, vol. $35, \mathrm{n}^{\circ} 4$, p. 1916-1957.

Ménard C. (2008), « A New Institutional Approach to Organization », in Ménard C. \& M. M. Shirley, Handbook of New Institutional Economics, Berlin, Springer, p. 281-318.

Meunier F. (2007), « Les hauts salaires dans la banque : talent ou collusion? », Revue française d'économie, vol. 22, n 1, p. 49-71.

Milgrom P. \& J. Robert (2003), Économie, organisation et management, Bruxelles, de boeck Université, traduit sous la direction de Bernard Ruffieux.

Mirallès P. (2007), « La gestion des talents : émergence d'un nouveau modèle de management ? », Management \& Avenir, vol. 11, n 1, p. 29-42.

Paché G. \& G. N'Goala (2011), « Les stratégies de création et d'appropriation de la valeur dans un contexte d'incertitude majeure : le cas du football professionnel », Management \& Avenir, vol.46, n o 6 , p. 53-78.

Rajan R. G. \& L. Zingales (2001), « The firm as a dedicated hierachy: a theory of the origins and growth of the firm », The Quarterly Journal of Iconomics, vol. 136, n 3, p. 805-851.

Rogerson W. P. (1992), « Contractual Solutions to the Hold-Up Problem », The Review of Economic Studies, vol. 59, $\mathrm{n}^{\circ} 4$, p. 777-793.

Rosen S. (1981), «The economics of superstars », The American Economic Review, vol. 71, n 5, p. 845-858.

Rosen S. (1986), «Prizes and incentives in elimination tournaments », American Economic Review, vol. 76, n 4, p. 701-715.

Sanderson A. R. (2002), " The many dimensions of competitive balance », Journal of Sports Economics, vol. 3, $\mathrm{n}^{\circ}$ 2, p. 204-228.

Segal I. (1999), « Complexity and Renegotiation: A Foundation for Incomplete Contracts », The Review of Economic Studies, vol. 66, n 1, p. 57-82. 
Slichter S. (1950), « Notes on the structure of wages ", Review of economics and statistics, vol. 32, p. 80-91.

Tang M.-C. (2015), « Contract length, expected surplus, and specific investments: empirical evidence from the National Football League ", Journal of Sports Economics, vol. 16, n 3, p. 295-311.

Tirole J. (1988), « The multicontract organization », The Canadian Journal of Economics, vol. 21, $n^{\circ} 3$, p. 459-466.

Tullock G. (1980), « Efficient Rent Seeking », in Buchanan J., Tollison R. \& G Tullock (eds.), Toward a Theory of Rent Seeking Society, A\&M University Press, p. 97-112.

UEFA (2008-2016), Panorama du football européen. Rapport de benchmarking sur la procédure d'octroi de licence aux clubs, exercices 2008 à 2016, Union des associations européennes de football.

Wahl A. \& P. Lanfranchi (1995), Les footballeurs professionnels des années trente à nos jours, Paris, Hachette.

Williamson O. E. (1971), « The Vertical Integration of Production: Market Failure Considerations » , American Economic Review, vol. 61, n² 2, p. 112-123.

Williamson O. E. (1975), Markets and Hierarchies, Analysis and Antitrust Implications: a Study in the Economics of Internal Organization, New York, The free press.

Williamson O. E. (1985), The Economic Institutions of Capitalism, New York/London, The free press, New York.

\section{NOTES}

1. Jean Tirole (1988) évoque quant à lui le caractère « multicontractuel » de l'entreprise.

2. P. Joskow évoque également cette solution par l'intégration verticale dans le cas des relations entre les mines de charbon et les centrales thermiques électriques nord-américaines en expliquant que les premières sont souvent la propriété des secondes dès lors qu'il n'existe pas de contrats de longue durée entre les deux parties.

3. Sur ce concept de "patrimoine productif», cf. le numéro spécial d'Économie appliquée de décembre 2014 «Le patrimoine, d'un objet à un instrument d'analyse" (tome LXVII, n4) coordonné par Christian Barrère.

4. Les arrêts Malaja (2002), Kolpak (2003) et Simutenkov (2005) ont élargi la zone communautaire du marché européen des transferts dans les sports collectifs aux 77 pays ayant signé les accords de Cotonou de 2000.

5. La course à l'armement entre les clubs dans l'obtention des joueurs ayant entraîné une course à l'armement financier, les clubs sont confrontés à un besoin de financement constant en dépit de la mutation de leur structure de financement .

6. Précisons que, au-delà des trois facteurs de valorisation présentés ici, un joueur pèse aussi sur la valeur boursière d'un club côté. Ainsi, son départ peut avoir des conséquences sur le cours de l'action du club.

7. A contrario ils bénéficient des investissements spécifiques des clubs précédents lorsqu'ils achètent un joueur.

8. La condition de transférabilité des actifs est aussi vérifiée dans le football lorsque le départ d'un joueur entraîne celui d'autres joueurs. Il s'agit du risque de départ collectif . À titre d'exemple, au terme de la saison 2016/2017 durant laquelle l'AS Monaco a obtenu de très bonnes performances sportives (champion de France, finaliste de la Coupe de la Ligue et demi-finaliste de 
la Ligue des Champions), le club a subi les départs successifs de plusieurs cadres: Tiémoué Bakayoko, Kylian Mbappé, Benjamin Mendy ou encore Bernardo Silva.

9. La réputation des joueurs se construit notamment au gré de leurs expériences professionnelles dans les clubs. Les joueurs cherchent ainsi à valoriser leur capital humain via les investissements collectifs des clubs dans le but de développer leurs "avantages compétitifs individuels " (Mirallès 2007).

10. Les stratégies de bluff sont parties intégrantes du problème du hold-up (e.g. Lyon \& Rasmusen 2004 ; Segal 1999 ; Rogerson 1992).

11. http://mercato.eurosport.fr/football/ligue-1/2015-2016/mercato-thiago-motta-reste-au-psget-prolonge-son-contrat-d-un-an_sto4882734/story.shtml, consulté le 29 septembre 2015.

12. S. Ramos a par ailleurs été nommé capitaine de l'équipe madrilène.

13. Après la signature du nouveau contrat de T. Motta, le Président du PSG, Nasser Al-Khelaiffi, a rappelé que le joueur était indispensable pour le club : "Thiago est un joueur que nos fans adorent, comme nous avons encore pu le mesurer lors de notre dernier match au Parc des Princes, où son nom fut scandé plusieurs fois. Depuis le début de la saison, il prouve à nouveau à quel point il reste un rouage essentiel de notre système de jeu. Ces dernières années, son immense expérience a beaucoup aidé à faire progresser son équipe. Son vécu en Champions League, qu'il a déjà remportée deux fois, va à nouveau nourrir notre ambition de franchir un cap au niveau européen. À mes yeux, il était hors de question de se séparer de ce très grand joueur $»$.

http://www.psg.fr/fr/News/003001/Article/72025/Thiago-Motta-prolonge-jusqu-en-2017, consulté le 29 septembre 2015.

14. Les revenus des clubs européens de première division ont augmenté de près de $414 \%$ entre 1997 et 2016 alors que leur masse salariale a progressé de près de $539 \%$ sur la même période.

15. Les revenus des clubs français de première division ont augmenté de près de $407 \%$ entre 1997 et 2016 alors que leur masse salariale a progressé de près de $472 \%$ sur la même période.

16. Ce que démontrent notamment Eberhard Feess et al. dans le cas de la Bundesliga sur la période allant de la saison 1996/97 à la saison 1999/2000.

17. À la même période, la Fédération Internationale de Football Association (FIFA) a instauré une indemnité de formation. Elle intervient quand un club fait signer un premier contrat professionnel à un joueur ainsi qu'à chaque transfert d'un joueur professionnel jusqu'à l'âge de 23 ans. Elle est acquittée par le club acheteur vers le ou les clubs ayant formé le joueur entre 12 et 23 ans. Les indemnités de formation ont pour but de couvrir les coûts de formation des joueurs. Par exemple, lors du transfert de C. Ronaldo de Manchester United au Real Madrid pour 94 millions d'euros, le Sporting Portugal a perçu 2,35 millions d'euros au titre de club formateur (Ronaldo y ayant évolué de 11 à 18 ans). L'instauration de cette indemnité de formation a peu incité les clubs à s'investir dans la formation de jeunes joueurs, sauf ceux pour lesquels la vente de joueurs est fondamentale dans leur budget. Dans ce dernier cas, il y a effet d'aubaine dans la mesure où ces clubs auraient de toute façon formé des joueurs pour les revendre. Par conséquent, parce qu'ils profiteront à terme à des concurrents, les clubs sont peu incités à investir dans la formation de jeunes joueurs .

18. D'une part, il est difficile d'évaluer la contribution sportive individuelle d'un joueur à un collectif. D'autre part, il est complexe de déterminer la contribution d'un joueur aux revenus globaux d'un club.

19. Il est intéressant de souligner que, en réaction à ce transfert, plusieurs "grands " clubs européens ont augmenté le niveau de la clause libératoire de leurs principaux joueurs. Jusqu'alors fixée à 250 millions d'euros, les dirigeants barcelonais ont par exemple revalorisé le montant de la clause libératoire de L. Messi pour que celle-ci atteigne 700 millions d'euros. C'est également le montant des clauses libératoires incluses aux contrats d'Isco et de Marco Asensio après renégociations contractuelles engagées par le Real Madrid en août et en septembre 2017. 
20. En tenant compte de l'ensemble des dettes et des arriérés de paiement des clubs, l'endettement global de la première division européenne s'élevait à 12 milliards d'euros en moyenne sur la période 2008-2012 (Bastien 2017), l'UEFA ne communiquant pas de données supplémentaires à cet effet après l'exercice 2012.

\title{
RÉSUMÉS
}

Partant du double constat de l'augmentation des salaires des joueurs et de la récurrence des déficits des clubs, l'objectif de cet article est de déterminer les mécanismes par lesquels les joueurs sont en mesure de capter les richesses du football professionnel en Europe. Dans cette perspective, l'article propose une application originale du «problème du hold-up » dont le but est de caractériser le fort pouvoir de négociation instauré par les joueurs dans leur relation contractuelle aux clubs. Ces derniers, pour qui les effets du "hold-up» se traduisent essentiellement par une hausse des charges salariales, développent des stratégies de résistance qui mènent à l'allongement de la durée des contrats et surtout à la hausse des indemnités de transfert. Paradoxalement, ces réactions contribuent à accroître les difficultés financières des clubs.

For decades in the European professional football, the players' wages have been increasing while the clubs have been piling up deficits. The goal of this article is to demonstrate that in professional football the wealth sharing is to the benefit the players. In this regard, this article aims to characterise the players' bargaining power against the clubs in their contractual relationship. For this purpose, we frame the bargaining between the players and the clubs in terms of the " hold-up problem ». For the clubs, this situation leads to the rise of salary expenses. That is why they develop strategies to minimize the hold-up effects: the extension of contracts duration and most importantly the increase of the transfer fee levels. Paradoxically, we show that these reactions extend the financial distresses of clubs.

\section{INDEX}

Mots-clés : contrat de travail, problème du hold-up, football

Keywords : labor contract, hold-up problem, football

\author{
AUTEUR \\ JÉRÉMIE BASTIEN \\ Chargé d'études économiques, Centre de de Droit et d'Economie du Sport (CDES), Limoges ; \\ jrm.bastien@gmail.com
}

